\title{
Highly Sensitive Electrostatic Force Detection Using Small Amplitude Frequency-Modulation Atomic Force Microscopy in the Second Flexural Mode*
}

\author{
Keisuke Nishi and Yoshihiro Hosokawa \\ Department of Electronic Science \& Engineering, \\ Kyoto University, Katsura, Nishikyo, Kyoto 615-8510, Japan, \\ Kei Kobayashi \\ Office of Society-Academia Collaboration for Innovation, \\ Kyoto University, Nishikyo, Kyoto 615-8520, Japan \\ Kazumi Matsushige and Hirofumi Yamada ${ }^{\dagger}$ \\ Department of Electronic Science \& Engineering, \\ Kyoto University, Katsura, Nishikyo, Kyoto 615-8510, Japan
}

(Received 1 December 2010; Accepted 25 January 2011; Published 2 April 2011)

\begin{abstract}
We propose a novel scheme of highly sensitive electrostatic force detection using a small oscillation amplitude of a cantilever in the second flexural mode of frequency-modulation atomic force microscopy, which is useful for Kelvinprobe force microscopy (KFM) and electrostatic force microscopy (EFM). In this novel scheme, the cantilever is mechanically oscillated at the second flexural resonance frequency with a small oscillation amplitude, while the electrostatic force detection is carried out at the first flexural resonance frequency. Because of the reduced average tip-sample distance and the low spring constant and high quality factor of the first flexural mode, the sensitivity for electrostatic force detection becomes very high. We calculated signal-to-noise ratios (SNRs) for the proposed scheme and conventional schemes, and found that the SNR of the proposed scheme is higher than that of conventional schemes. We also performed KFM/EFM measurements using the proposed and conventional schemes on metal phthalocyanine thin films.
\end{abstract}

[DOI: $10.1380 /$ ejssnt.2011.146]

Keywords: Atomic force microscopy; Surface electronic phenomena; Nano-scale imaging, measurement, and manipulation technology; Frequency-modulation atomic force microscopy; Kelvin-probe force microscopy; Small oscillation amplitude; second flexural mode

\section{INTRODUCTION}

Frequency-modulation atomic force microscopy (FMAFM) is a powerful imaging tool with a high force sensitivity $[1,2]$. In FM-AFM, the interaction force between the tip and the sample is detected as a frequency shift of the cantilever when the cantilever is oscillated at its resonance frequency. In general, interaction forces between the tip and the sample surface can be categorized by their decay length as short-range forces such as chemical bonding forces or long-range forces such as van der Waals and electrostatic forces. Since the decay lengths of the short-range forces are on the order of interatomic or intermolecular distances, it is possible to achieve atomic or molecular resolution by FM-AFM.

Kelvin-probe force microscopy (KFM), which is capable of mapping the surface potential of a sample using dynamic-mode AFM [3, 4], can be also implemented using FM-AFM. Two schemes have been proposed to implement KFM using FM-AFM. Kikukawa et al. proposed the detection of electrostatic forces with the second flexural mode, while the tip-sample distance is regulated using the first flexural mode [5]. In this scheme, a bias modula-

\footnotetext{
* This paper was presented at the 13th International Conference on Non-Contact Atomic Force Microscopy (NC-AFM2010), Ishikawa Ongakudo, Kanazawa, Japan, 31 July-4 August, 2010.

†Corresponding author: h-yamada@kuee.kyoto-u.ac.jp
}

tion voltage with the second flexural resonance frequency is applied between the tip and sample, and the modulation component of the cantilever displacement signal is detected (2nd-AM-KFM). On the other hand, Kitamura et al. developed a scheme to detect a modulated frequency shift component induced by applying a modulation bias voltage with a frequency lower than the bandwidth of the frequency demodulator [6]. In this scheme, both electrostatic force detection and tip-sample distance regulation are carried out in the first flexural mode (1st-FM-KFM). Both schemes are sensitive enough to achieve atomic resolution in surface potential images [6-8].

Recent studies have shown that the oscillation amplitude of the cantilever, and thereby the average tip-sample distance can be reduced by using very-high spring constant cantilevers $[9,10]$. The oscillation may become unstable, or the tip may jump into a static contact if a cantilever with a low spring constant is used. Kawai et al. successfully reduced the oscillation amplitude to less than $0.1 \mathrm{~nm}$ by using a high effective spring constant in the second flexural mode. We also succeeded in achieving molecular-resolution imaging of lead phthalocyanine $(\mathrm{PbPc})$ deposited on an $\mathrm{MoS}_{2}$ surface using the second flexural mode with an oscillation amplitude of $0.5 \mathrm{~nm}$ [11]. Since the average tip-sample distance is small in smallamplitude FM-AFM utilizing the second flexural mode (2nd-FM-AFM), an increase in the sensitivity of electrostatic force detection can also be expected.

In this paper, we propose a novel scheme of highlysensitive electrostatic force detection with 2nd-FM-AFM 
TABLE I: Summary of possible schemes for electrostatic force detection using FM-AFM. 2nd-AM-KFM and 1st-FM-KFM are based on conventional FM-AFM, while SA-AM-KFM and SA-FM-KFM are based on small-amplitude FM-AFM.

\begin{tabular}{lllll}
\hline \hline Scheme & Flexural mode for topography & Frequency of bias modulation & Modulation of electrostatic force & Reference \\
\hline 2nd-AM-KFM & 1st & 2nd & AM & {$[12]$} \\
1st-FM-KFM & 1st & low frequency $(\sim 1 \mathrm{kHz})$ & FM & {$[11]$} \\
SA-AM-KFM & 2nd (small amplitude) & 1st & AM & \\
SA-FM-KFM & 2nd (small amplitude) & low frequency $(\sim 1 \mathrm{kHz})$ & FM & \\
\hline \hline
\end{tabular}

for KFM and electrostatic force microscopy (EFM). In this novel scheme, a bias modulation voltage at the first flexural resonance frequency is applied between the tip and sample, and the modulation component of the cantilever displacement signal is detected. We calculated signal-to-noise ratios (SNRs) for the proposed scheme and conventional schemes, and found that the SNR of the proposed method is higher than that of conventional methods. We also performed KFM/EFM measurements using the proposed and conventional methods on metal phthalocyanine thin films.

\section{KFM USING SMALL OSCILLATION AMPLITUDE FM-AFM IN THE SECOND FLEXURAL MODE}

There are two possible schemes for the detection of electrostatic forces by 2nd-FM-AFM [10, 11]: AM-KFM in the first flexural mode, and FM-KFM in the second flexural mode. Since the first flexural mode is not utilized in 2nd-FM-AFM, the modulation component in the cantilever deflection signal can be detected if a bias modulation voltage with the first flexural resonance frequency is applied between the tip and the sample (SA-AM-KFM). On the other hand, the FM-KFM scheme can be applied to the second flexural mode. In this scheme, both elec- trostatic force detection and tip-sample distance regulation are carried out in the second flexural mode (SA-FMKFM). Table I summarizes possible schemes for electrostatic force detection using FM-AFM.

Since the stiffness of the cantilevers commonly used for conventional FM-AFM is on the order of $10 \mathrm{~N} / \mathrm{m}$, the oscillation amplitude at the first flexural mode is typically larger than $5 \mathrm{~nm}$. However, in the 2nd-FM-AFM, the oscillation amplitude can be safely reduced to less than $1 \mathrm{~nm}$ because of the high effective spring constant of the second flexural mode. Since the average tip-sample distance is smaller than in the two schemes based on conventional FM-AFM, enhanced sensitivity of electrostatic force detection can be expected for SA-KFM schemes (SA-AMKFM and SA-FM-KFM) based on the 2nd-FM-AFM, as listed in Table I. In the following discussion, we calculate the SNR for each scheme listed in Table I.

We calculated SNRs for EFM in a model system by each scheme, as the magnitude of the modulated signal, induced by a given modulation bias voltage with an offset, divided by that of the noise. The model system was a cantilever having a conical tip with a semispherical apex and a planar surface. The electrostatic force between the tip and the plane $\left(F_{\text {tip }(0)}\right)$ induced by applying a voltage $(V)$ as a function of $z$, which was the distance between the apex and the plane, was given by Hudlet et al. as

$$
F_{\mathrm{tip}(0)}=\pi \varepsilon_{0} V^{2}\left[\frac{r^{2}(1-\sin \theta)}{z(z+r(1-\sin \theta))}+\frac{1}{(\ln \tan (\theta / 2))^{2}}\left(\ln \frac{h}{z+r(1-\sin \theta)}-1+\frac{r \cos ^{2} \theta / \sin \theta}{(z+r(1-\sin \theta))}\right)\right]
$$

where $\varepsilon_{0}$ is the dielectric constant in vacuum. $r, \theta$, and $h$ are the radius of the tip, half the cone angle, and the height of the cone, respectively [12]. We also took into account the uniform electrostatic force exerted on the cantilever. If we consider a cantilever with an infinitesimal length and the sample as a parallel plate system, the electrostatic force per unit length $\left(d F_{\text {cantilever(0) }}\right)$ is given by

$$
d F_{\text {cantilever }(0)}=\frac{w}{2 h^{2}} \varepsilon_{0} V^{2},
$$

where $w$ is the width of the cantilever. Since the displacement of the cantilever end caused by the uniformly distributed force of $d F_{\text {cantilever(0) }}$ is $3 / 8$ of that caused by the concentrated force of $d F_{\text {cantilever(0) }} \cdot l$ at the end [13], we assumed that the effective force exerted on the tip by the uniformly distributed electrostatic force between the cantilever and the plane was

$$
F_{\text {cantilever(0) }}=\frac{3}{8} d F_{\text {cantilever }(0)} \cdot l=\frac{3 w l}{16 h^{2}} \varepsilon_{0} V^{2}
$$

where $l$ is the length of the cantilever. Note that we assumed that the force on the cantilever did not depend on $z$, because $h$ is much larger than $z$. When a modulation bias voltage of amplitude $V_{\mathrm{ac}}$ and modulation frequency $f_{\mathrm{m}}$ is applied with an offset voltage of $V_{\mathrm{dc}}$ as $V=V_{\mathrm{dc}}+V_{\mathrm{ac}} \cos 2 \pi f_{\mathrm{m}} t$, the total force component oscillating at $f_{\mathrm{m}}$ becomes 


$$
\begin{aligned}
F_{\text {total }}= & \left(F_{\mathrm{tip}(\mathrm{m})}+F_{\text {cantilever }(\mathrm{m})}\right) \cos 2 \pi f_{\mathrm{m}} t \\
= & 2 \pi \varepsilon_{0} V_{\mathrm{dc}} V_{\mathrm{ac}}\left[\frac{r^{2}(1-\sin \theta)}{z(z+r(1-\sin \theta))}+\frac{1}{(\ln \tan (\theta / 2))^{2}}\left(\ln \frac{h}{z+r(1-\sin \theta)}-1+\frac{r \cos ^{2} \theta / \sin \theta}{(z+r(1-\sin \theta))}\right)\right] \cos 2 \pi f_{\mathrm{m}} t \\
& +\frac{3 w l}{8 h^{2}} \varepsilon_{0} V_{\mathrm{dc}} V_{\mathrm{ac}} \cos 2 \pi f_{\mathrm{m}} t .
\end{aligned}
$$

First, we calculated the SNR for 2nd-AM-KFM. In this scheme, the tip oscillates with an amplitude $A_{1}$ at the first flexural resonance frequency $f_{1}$ as $z=z_{0}+A_{1}(1+$ $\left.\cos 2 \pi f_{1} t\right)=z_{0}+A_{1}(1+u), z_{0}$ being the closest apexplane distance, while the modulation frequency $f_{\mathrm{m}}$ is set at $f_{2}$. The electrostatic force acting on the cantilever at $f_{2}$ is averaged over a period of $1 / f_{1}$ since $f_{1}$ and $f_{2}$ are not commensurate. Therefore the deflection caused by the total force at $f_{2}$ can be described as

$$
\begin{aligned}
\Delta d_{2 \mathrm{nd}-\mathrm{AM}-\mathrm{KFM}} & =\frac{Q_{2}}{k_{2}}\left\langle F_{\text {total }(\mathrm{m})}\right\rangle \cos 2 \pi f_{2} t \\
& =\frac{Q_{2}}{k_{2}}\left(f_{1} \int_{0}^{1 / f_{1}} F_{\text {total }(\mathrm{m})} d t\right) \cos 2 \pi f_{2} t \\
& =\frac{Q_{2}}{k_{2}}\left(\frac{1}{\pi} \int_{-1}^{1} \frac{F_{\text {total }(\mathrm{m})}}{\sqrt{1-u^{2}}} d u\right) \cos 2 \pi f_{2} t
\end{aligned}
$$

where $k_{2}$ and $Q_{2}$ are the effective spring constant and quality factor of the second flexural mode, respectively. If we detect the modulated deflection using a displacement sensor with a sensitivity of $s_{\mathrm{ds}}$, the root-mean-squared amplitude of the modulated signal becomes

$$
S_{2 \text { nd-AM-KFM }}=\frac{s_{\mathrm{ds}}}{\sqrt{2}} \frac{Q_{2}}{k_{2}}\left\langle F_{\text {total }(\mathrm{m})}\right\rangle .
$$

When the modulated signal is detected by a lock-in am- plifier with measurement bandwidth $B$, the magnitude of noise in the output of the lock-in amplifier can be obtained by integrating the displacement noise density for the frequency range of $f_{2} \pm B$ as

$$
N_{2 \mathrm{nd}-\mathrm{AM}-\mathrm{KFM}}=s_{\mathrm{ds}} \sqrt{\int_{f_{2}-B}^{f_{2}+B}\left(n_{2 \mathrm{nd}-\mathrm{th}}^{2}+\left({\frac{n_{\mathrm{ds}}}{3.5}}^{2}\right)\right) d f}
$$

as shown in a schematic of the frequency spectrum in Fig. 1(a), where $n_{2 \text { nd }- \text { th }}$ and $n_{\text {ds }}$ are the thermal displacement noise density and noise-equivalent displacement density of the displacement sensor, respectively. We assumed that the displacement sensor was an optical beam deflection sensor, and assumed a reduction of the noiseequivalent displacement density at $f_{2}$ by a factor of 3.5 , since the angle of the cantilever end for a given displacement at $f_{2}$ becomes 3.5 times that of the same static displacement in an ideal case [11]. The thermal noise density for the second flexural mode $n_{2 n d-t h}$ is given by

$$
n_{2 \text { nd }- \text { th }}=\sqrt{\frac{2 k_{\mathrm{B}} T}{\pi k_{2} f_{2} Q_{2}} \frac{1}{\left(1-\left(f / f_{2}\right)^{2}\right)^{2}+\left(f /\left(f_{2} Q_{2}\right)\right)^{2}}}
$$

where $k_{\mathrm{B}}$ is the thermal energy [14], and we obtain an SNR for 2nd-AM-KFM as

$$
S N R_{2 \mathrm{nd}-\mathrm{AM}-\mathrm{KFM}}=\frac{S_{2 \mathrm{nd}-\mathrm{AM}-\mathrm{KFM}}}{N_{2 \mathrm{nd}-\mathrm{AM}-\mathrm{KFM}}}=\frac{Q_{2}\left\langle F_{\mathrm{total}(\mathrm{m})}\right\rangle}{\sqrt{2} k_{2}}\left(\int_{f_{2}-B}^{f_{2}+B}\left(n_{2 \mathrm{nd}-\mathrm{th}}^{2}+\left(\frac{n_{\mathrm{ds}}}{3.5}\right)^{2}\right) d f\right)^{-1 / 2}
$$

Secondly, we calculated an SNR for 1st-FM-KFM. In this scheme, a bias voltage with a modulation frequency sufficiently lower than the bandwidth of the frequency demodulator was applied while the tip was oscillating with an amplitude of $A_{1}$ at $f_{1}$. The modulated frequency shift component induced by the modulation bias voltage is given by

$$
\Delta f_{1 \mathrm{st}-\mathrm{FM}-\mathrm{KFM}}=-\frac{f_{1}}{k_{1} A_{1}^{2}}\left\langle F_{\mathrm{total}} \cdot A_{1} \cos 2 \pi f_{1} t\right\rangle=-\frac{f_{1}}{k_{1} A_{1}^{2}}\left\langle F_{\mathrm{tip}(\mathrm{m})} \cdot A_{1} \cos 2 \pi f_{1} t\right\rangle \cos 2 \pi f_{\mathrm{m}} t
$$

where $k_{1}$ is the spring constant of the first flexural mode [15]. Note that the force between the cantilever and plane does not contribute to the frequency shift. This equation reduces to 


$$
\begin{aligned}
\Delta f_{1 \mathrm{st}-\mathrm{FM}-\mathrm{KFM}} & =-\frac{f_{1}}{k_{1} A_{1}^{2}}\left\langle f_{1} \int_{0}^{1 / f_{1}}\left(F_{\mathrm{tip}(\mathrm{m})} \cdot A_{1} \cos 2 \pi f_{1} t\right) d t\right\rangle \cos 2 \pi f_{\mathrm{m}} t \\
& =-\frac{f_{1}}{k_{1} A_{1}}\left\langle\frac{1}{\pi} \int_{-1}^{1} F_{\mathrm{tip}(\mathrm{m})} \frac{u}{\sqrt{1-u^{2}}} d u\right\rangle \cos 2 \pi f_{\mathrm{m}} t .
\end{aligned}
$$

The frequency spectrum in 1st-FM-KFM is shown in Fig. 1(b), in which two sidebands appear at $f_{1} \pm f_{\mathrm{m}}$. When the modulated signal is detected by the frequency demodulator and the lock-in amplifier with measurement bandwidth $B$, the magnitude of noise in the output of the lock-in amplifier is given by integrating the frequency noise density over the frequency range of $f_{\mathrm{m}} \pm B$ as [16]

$$
\delta f_{1 \mathrm{st}-\mathrm{FM}-\mathrm{KFM}}=\sqrt{\int_{f_{\mathrm{m}}-B}^{f_{\mathrm{m}}+B}\left(\frac{f_{1} k_{\mathrm{B}} T}{\pi k_{1} Q_{1} A_{1}^{2}}+\frac{2 n_{\mathrm{ds}}^{2}}{A_{1}^{2}} f^{2}\right) d f .}
$$

The SNR for 1st-FM-KFM becomes

$$
S N R_{1 \mathrm{st}-\mathrm{FM}-\mathrm{KFM}}=-\frac{f_{1}\left\langle F_{\mathrm{tip}(\mathrm{m})} \cdot A_{1} \cos 2 \pi f_{1} t\right\rangle}{\sqrt{2} k_{1} A_{1}^{2}}\left(\frac{2 f_{1} k_{\mathrm{B}} T B}{\pi k_{1} Q_{1} A_{1}^{2}}+\frac{4 n_{\mathrm{ds}}^{2} B^{3}}{3 A_{1}^{2}}+\frac{4 n_{\mathrm{ds}}^{2} B}{A_{1}^{2}} f_{\mathrm{m}}^{2}\right)^{-1 / 2} .
$$

Finally, we calculated SNRs for SA-AM-KFM and SAFM-KFM. In SA-AM-KFM, the tip oscillates with an amplitude of $A_{2}$ at $f_{2}$, while the modulation frequency $f_{\mathrm{m}}$ was set to $f_{1}$, as shown in the frequency spectrum in Fig. 1(c). The deflection caused by the total force at $f_{1}$ becomes

$$
\begin{aligned}
\Delta d_{\mathrm{SA}-\mathrm{AM}-\mathrm{KFM}} & =\frac{Q_{1}}{k_{1}}\left\langle F_{\text {total }(\mathrm{m})}\right\rangle \cos 2 \pi f_{1} t \\
& =\frac{Q_{1}}{k_{1}}\left(f_{2} \int_{0}^{1 / f_{2}} F_{\text {total }(\mathrm{m})} d t\right) \cos 2 \pi f_{1} t \\
& =\frac{Q_{1}}{k_{1}}\left(\frac{1}{\pi} \int_{-1}^{1} \frac{F_{\text {total }(\mathrm{m})}}{\sqrt{1-u^{2}}} d u\right) \cos 2 \pi f_{1} t
\end{aligned}
$$

Therefore, the SNR for SA-AM-KFM becomes

in the same manner as in Eq. (5). The root-mean-squared amplitude of the modulated signal becomes

$$
S N R_{\mathrm{SA}-\mathrm{AM}-\mathrm{KFM}}=\frac{Q_{1}\left\langle F_{\mathrm{total}(\mathrm{m})}\right\rangle}{\sqrt{2} k_{1}}\left(\int_{f_{1}-B}^{f_{1}+B}\left(n_{1 \mathrm{st}-\mathrm{th}}^{2}+n_{\mathrm{ds}}^{2}\right) d f\right)^{-1 / 2}
$$

in the same manner as in Eq. (9). $n_{1 \mathrm{st}-\mathrm{th}}$ is the thermal noise density of the first flexural mode,

$$
n_{1 \mathrm{st}-\mathrm{th}}=\sqrt{\frac{2 k_{\mathrm{B}} T}{\pi k_{1} f_{1} Q_{1}} \frac{1}{\left(1-\left(f / f_{1}\right)^{2}\right)^{2}+\left(f /\left(f_{1} Q_{1}\right)\right)^{2}}} .
$$

On the other hand, in SA-FM-KFM, as shown in the frequency spectrum in Fig. 1(d), a bias voltage with a low modulation frequency was applied. Two sidebands appeared at $f_{2} \pm f_{\mathrm{m}}$, and the SNR for SA-FM-KFM becomes

$$
S N R_{\mathrm{SA}-\mathrm{FM}-\mathrm{KFM}}=-\frac{f_{2}\left\langle F_{\mathrm{tip}(\mathrm{m})} \cdot A_{2} \cos 2 \pi f_{2} t\right\rangle}{\sqrt{2} k_{2} A_{2}^{2}}\left(\frac{2 f_{2} k_{\mathrm{B}} T B}{\pi k_{2} Q A_{2}^{2}}+\frac{4 n_{\mathrm{ds}}^{2} B^{3}}{3 \cdot 3.5^{2} A_{2}^{2}}+\frac{4 n_{\mathrm{ds}}^{2} B}{3.5^{2} A_{2}^{2}} f_{m}^{2}\right)^{-1 / 2}
$$

in the same manner as in Eq. (13).

We evaluated the SNR for each scheme using practi- 

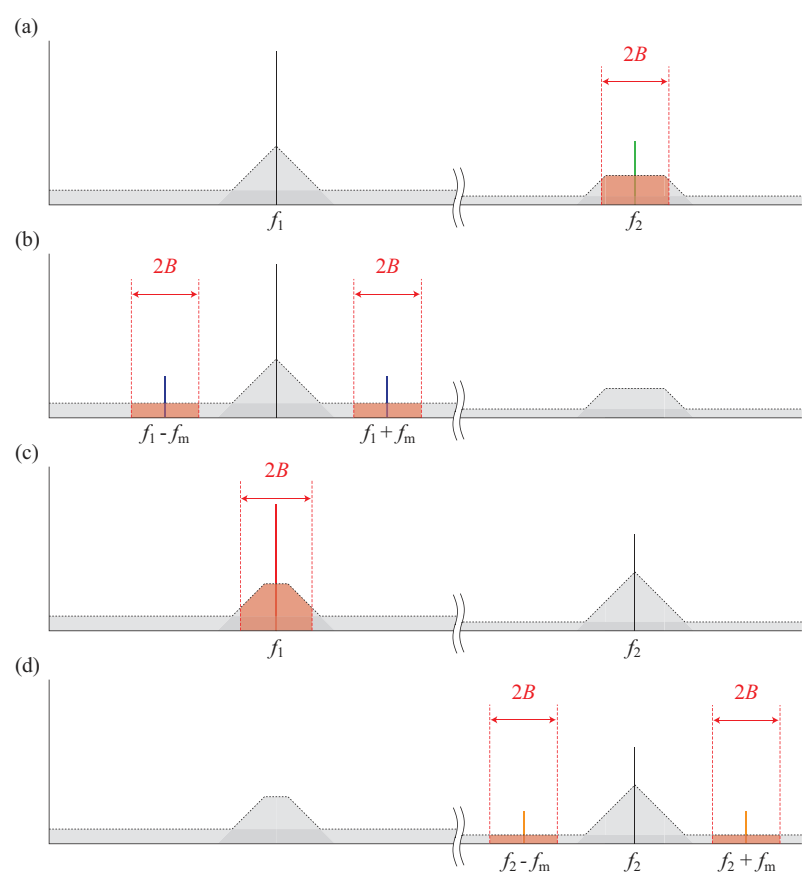

FIG. 1: Schematic illustration of frequency spectra of cantilever displacement in FM-AFM with modulation components induced by a modulation bias voltage at $f_{\mathrm{m}}$. (a) Conventional AM-KFM using the second flexural mode (2nd-AM-KFM). (b) Conventional FM-KFM using the first flexural mode (1st-FMKFM). (c) Small amplitude AM-KFM (SA-AM-KFM) and (d) small amplitude FM-KFM (SA-FM-KFM). Two side bands appear in the FM-KFM schemes. The frequency range for integration of the noise determined by the measurement bandwidth $B$ is indicated in each spectrum.

cal experimental parameters. We used $V_{\mathrm{dc}}=0.01 \mathrm{~V}$, $V_{\mathrm{ac}}=1 \mathrm{~V}, z_{0}=0.3 \mathrm{~nm}, l=125 \mu \mathrm{m}, w=30 \mu \mathrm{m}$, $r=10 \mathrm{~nm}, \theta=25^{\circ}, h=10 \mu \mathrm{m}, k_{1}=40 \mathrm{~N} / \mathrm{m}, f_{1}=$ $300 \mathrm{kHz}, Q_{1}=30,000, k_{2}=1,600 \mathrm{~N} / \mathrm{m}, f_{2}=1.8 \mathrm{MHz}$, $Q_{2}=10,000, T=300 \mathrm{~K}, n_{\mathrm{ds}}=40 \mathrm{fm} / \sqrt{\mathrm{Hz}}$, and $B=100 \mathrm{~Hz}$. The modulation frequency $f_{\mathrm{m}}$ for 1st-FMKFM and SA-FM-KFM was set to $1 \mathrm{kHz}$. Figure 2 shows plots of the SNR for each method as a function of oscillation amplitude of the flexural mode used for tip-sample distance regulation. SNRs for the conventional schemes were consistent to a similar analysis recently published by Kawai et al. [8]. Assuming that a typical oscillation amplitude in FM-AFM using the first flexural mode is $10 \mathrm{~nm}$, and that $1 \mathrm{~nm}$ is typical of $2 \mathrm{nd}-\mathrm{FM}-\mathrm{AFM}$, we can compare SNRs of the conventional schemes at an oscillation amplitude of $10 \mathrm{~nm}$ and SA-KFM schemes at $1 \mathrm{~nm}$, as indicated by the arrows in Fig. 3. As a result, the SNR of SA-AMKFM is the highest of all schemes, mainly because of the reduction of the average tip-sample distance by the use of the second flexural resonance mode, and because of the smaller spring constant and higher quality factor of the first flexural mode than of the second. It should be noted that in SA-KFM schemes, the tip-sample distance is typically small, and applying a large modulation bias voltage induces a significant electrostatic force, which deteriorates the resolution of the topographic image. Therefore, in practice, one may set $V_{\text {ac }}$ lower than in conventional schemes, which reduces the SNRs of SA-KFM schemes.

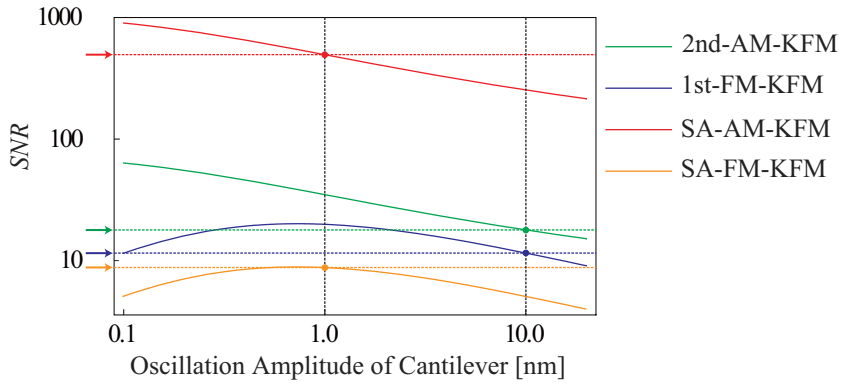

FIG. 2: Calculated signal-to-noise ratios of possible schemes for electrostatic force detection using FM-AFM. Typical oscillation amplitudes in 2nd-AM-KFM and 1st-FM-KFM are $10 \mathrm{~nm}$ for stable self-oscillation of the cantilever. On the other hand, the oscillation amplitude can be safely reduced to the order of $1 \mathrm{~nm}$ for schemes based on 2nd-FM-AFM. The arrows indicate the SNR at a typical oscillation amplitude for each scheme.

Since the electrostatic force between the cantilever and the sample surface is detected in AM-KFM schemes, the surface potential measured by AM-KFM schemes is averaged over a larger area than that measured by FM-KFM schemes [17]. It should also be mentioned that this effect is reduced in SA-AM-KFM because of the small amplitude operation. The following section describes the experimental results of surface potential measurements using the SA-AM-KFM scheme.

\section{EXPERIMENTAL RESULTS AND DISCUSSION}

We performed surface potential measurements on metal phthalocyanine molecular thin films using 1st-FM-KFM and SA-AM-KFM schemes. All measurements were performed in a ultrahigh-vacuum conditions at a typical base pressure of $3 \times 10^{-7} \mathrm{~Pa}$ using a commercially available AFM apparatus (JEOL JAFM-4500XT). Several optical components and electronics, such as a laser diode, a collimator, a focusing lens, and preamplifiers were replaced in order to achieve a low deflection sensor noise and a wide bandwidth [18]. A home-built FM controller [19] was used to oscillate the cantilever at its resonance frequency and to detect the frequency shift using a phase-locked loop (PLL) circuit. We used Si cantilevers (Nanoworld NCH, highly-doped, uncoated) whose nominal spring constant was $40 \mathrm{~N} / \mathrm{m}$. An $\mathrm{MoS}_{2}$ substrate was cleaved and subsequently introduced into the vacuum chamber. The substrate was prebaked at $200^{\circ} \mathrm{C}$ for 1 hour prior to the deposition of the molecules.

Figure 3 shows a schematic diagram of the SA-AMKFM. In the SA-AM-KFM, the cantilever is oscillated at $f_{2}$ for tip-sample distance regulation, while the surface potential measurement was carried out using the first flexural mode. $f_{1}, f_{2}, Q_{1}$, and $Q_{2}$ were $300 \mathrm{kHz}, 1.9 \mathrm{MHz}$, 13,000, and 9,000 respectively. The noise-equivalent displacement density of the displacement sensor at $f_{1}$ and $f_{2}$ were $45 \mathrm{fm} / \sqrt{\mathrm{Hz}}$ and $18 \mathrm{fm} / \sqrt{\mathrm{Hz}}$, respectively. We used a fourth-order Chebyshev-type high-pass filter (HPF) and low-pass filter (LPF) to separate the signal components 


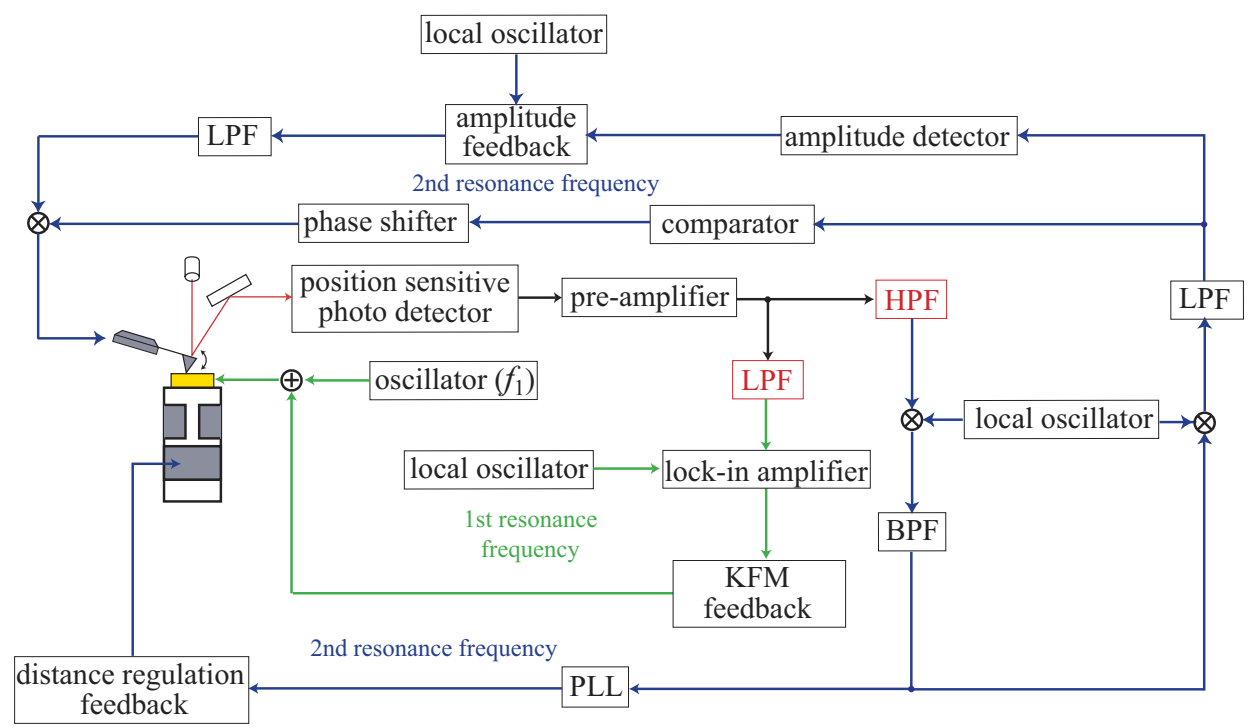

FIG. 3: Schematic diagram of the experimental setup for SA-AM-KFM. In this scheme, the cantilever was oscillated at its second flexural mode and the electrostatic force detection was carried out in the first resonance mode.

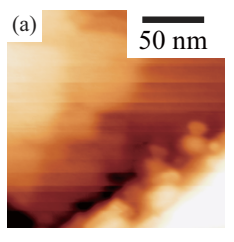

(d)
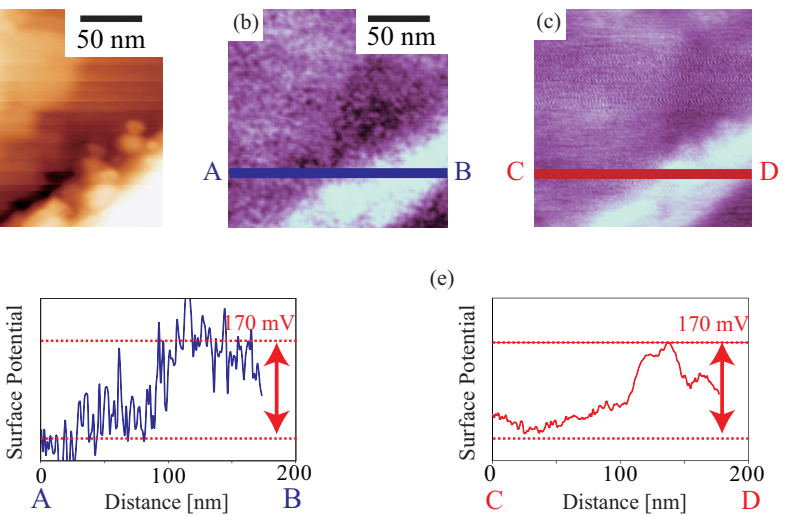

(e)

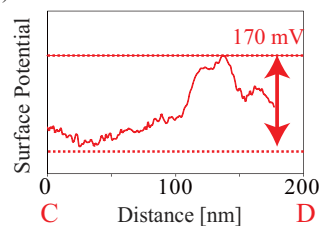

FIG. 4: (a) Topographic image and (b) surface potential image of $\mathrm{PbPc}$ thin film using conventional FM-KFM in the first flexural mode. (c) Surface potential image of the same area obtained using AM-KFM in the first flexural mode while the tip-sample distance was regulated with small-amplitude 2ndFM-AFM. (d) and (e) are cross-sectional profiles measured on the lines in (b) and (c), respectively.

for stable self-oscillation of the cantilever. An HPF with a cutoff frequency of $1 \mathrm{MHz}$ was used to pass the displacement signal components around $f_{2}$, while the LPF with a cutoff frequency of $730 \mathrm{kHz}$ was used to pass those around $f_{1}$. A local oscillator was used to convert $f_{2}$ to an intermediate frequency (IF) of $4.5 \mathrm{MHz}$, since the center frequency of the PLL was fixed at the IF. Another LPF was used to obtain an excitation frequency at $f_{2}$ [19]. For SA-AM-KFM, we set $f_{\mathrm{m}}$ and $V_{\mathrm{ac}}$ at $f_{1}$ and $50 \mathrm{mV}$, respectively. Note that since the frequency shift at $f_{2}$ was kept constant by the feedback electronics, the frequency shift at $f_{1}$ was also constant. We also performed KFM measurements using 1st-FM-KFM, in which we set $f_{\mathrm{m}}$ and $V_{\text {ac }}$ at $1 \mathrm{kHz}$ and $0.25 \mathrm{~V}$, respectively.

Figure 4 shows experimental results obtained using 1stFM-KFM and SA-AM-KFM. We deposited 4-5 molecular
Topography

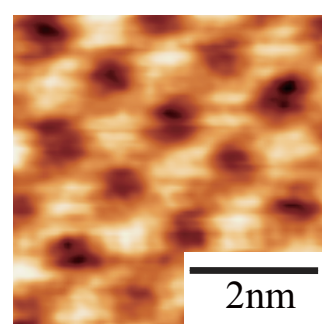

EFM

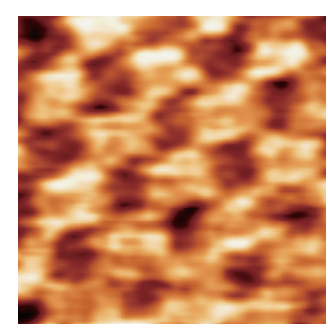

FIG. 5: Molecular-resolution topographic image and electrostatic force image of metal phthalocyanine thin film simultaneously obtained using SA-AM-KFM. The EFM image clearly shows a molecular-resolution contrast, even with the small modulation voltage of $15 \mathrm{mV}$.

layers (MLs) of $\mathrm{PbPc}$ molecules onto the $\mathrm{MoS}_{2}$ substrate at a rate of $0.03 \mathrm{ML} / \mathrm{min}$ while the substrate was kept at room temperature. Figures $4(\mathrm{a})$ and $4(\mathrm{~b})$ are a simultaneously acquired topographic image and surface potential image of the $\mathrm{PbPc}$ thin film using conventional 1st-FMKFM with an $A_{1}$ of $10 \mathrm{~nm}$, while Fig. 4(c) is a surface potential image acquired using the SA-AM-KFM scheme. In the SA-AM-KFM scheme, the tip-sample distance was regulated using the second flexural mode with an $A_{2}$ of $1.2 \mathrm{~nm}$. Aggregated $\mathrm{PbPc}$ molecules are visible in the lower right area of the topographic image, where a higher surface potential was detected in both Figs. 4(b) and 4(c). Cross-sectional line profiles measured on Figs. 4(b) and 4(c) are shown in Figs. 4(d) and (e), respectively, and indicate that the surface potential on the aggregates was about $170 \mathrm{mV}$ higher than the surrounding area. There was less noise in the surface potential and the corresponding profile taken by the SA-AM-KFM scheme than in those obtained by 1st-FM-KFM, even though the bias modulation amplitude was smaller.

Finally, we demonstrated molecular-resolution surface potential imaging using SA-AM-EFM. We deposited 
$\mathrm{PbPc}$ and copper phthalocyanine $(\mathrm{CuPc})$ molecules simultaneously onto the $\mathrm{MoS}_{2}$ substrate at a rate of $0.03 \mathrm{ML} / \mathrm{min}$, while the substrate was kept at room temperature. The thickness of the film was about 5 MLs. Figure 5(a) shows a topographic image of the metal phthalocyanine thin film using the second flexural mode with an oscillation amplitude of $1.2 \mathrm{~nm}$. Individual molecules are clearly resolved in the images, although we could not yet determine whether the resolved molecules were $\mathrm{PbPc}$ or $\mathrm{CuPc}$. During the generation of a topographic image by 2nd-FM-AFM, we applied $V_{\text {ac }}$ of $15 \mathrm{mV}$ at $f_{1}$, and the in-phase component at $f_{1}$ in the deflection signal was recorded as an electrostatic force image, as shown in Fig. 5(b), which clearly shows molecular-resolution contrast. The resolution was greatly improved compared to the surface potential image of the $\mathrm{CuPc}$ thin film in Ref. 20, which was obtained by conventional FM-KFM using the first flexural mode and a small modulation voltage.

\section{CONCLUSIONS}

We examined a novel scheme for KFM/EFM measurements with small oscillation amplitudes using the sec- ond flexural resonance mode of the cantilever. In this scheme, electrostatic force detection is carried out by detecting the modulated displacement at the first flexural resonance frequency (SA-AM-KFM). We calculated SNRs of conventional schemes and the novel scheme using practical experimental parameters, and showed that the SNR of SA-AM-KFM is much higher than that of the others because of the reduced average tip-sample distance, and lower spring constant and higher quality factor of the first flexural mode. We also performed surface potential measurements on metal phthalocyanine molecules using the SA-AM-KFM scheme, and showed an increase of the SNR compared to that of conventional FM-KFM using the first flexural mode.

\section{Acknowledgments}

This work was supported by Grants-in-Aid for Scientific Research from the Ministry of Education, Culture, Sports, Science and Technology of Japan and the Global COE Program of the Japanese Society for the Promotion of Science.
[1] T. R. Albrecht, P. Grutter, D. Home, and D. Rugar, J. Appl. Phys. 69, 668 (1991).

[2] S. Morita, R. Wiesendanger, and E. Meyer (Eds.), Noncontact Atomic Force Microscopy, 1st ed. (Springer, 2002), and references therein.

[3] J. M. R. Weaver and D. W. Abraham, J. Vac. Sci. Technol. B 9, 1559 (1991).

[4] M. Nonnenmacher, M. P. O'Boyle, and H. K. Wickramasinghe, Appl. Phys. Lett. 58, 2921 (1991).

[5] A. Kikukawa, S. Hosaka, and R. Imura, Rev. Sci. Instrum. 67, 1463 (1996).

[6] S. Kitamura and M. Iwatsuki, Appl. Phys. Lett. 72, 3154 (1998).

[7] T. Eguchi, Y. Fujikawa, K. Akiyama, T. An, M. Ono, T. Hashimoto, Y. Morikawa, K. Terakura, T. Sakurai, M. G. Lagally, and Y. Hasegawa, Phys. Rev. Lett. 93, 266102 (2004).

[8] S. Kawai, T. Glatzel, H.-J. Hug, and E. Meyer, Nanotechnology 21, 245704 (2010).

[9] F. J. Giessibl, Appl. Phys. Lett. 76, 1470 (2000).

[10] S. Kawai, S. Kitamura, D. Kobayashi, S. Meguro, and H.
Kawakatsu, Appl. Phys. Lett. 86, 193107 (2005).

[11] T. Ichii, Y. Hosokawa, K. Kobayashi, K. Matsushige, and H. Yamada, Appl. Phys. Lett. 94, 133110 (2009).

[12] S. Hudlet, M. Saint, C. Guthmann, and J. Berger, Euro. Phys. J. B 2, 5 (1998).

[13] W. C. Young and R. G. Budynas, Roark's Formulas for Stress and Strain, 7th ed. (McGraw-Hill, 2001).

[14] P. R. Saulson, Phys. Rev. D 42, 2437 (1990).

[15] F. J. Giessibl, Phys. Rev. B 56, 16010 (1997).

[16] K. Kobayashi, H. Yamada, and K. Matsushige, Rev. Sci. Instrum. 80, 043708 (2009).

[17] U. Zerweck, C. Loppacher, T. Otto, S. Grafstrom, and L. M. Eng, Phys. Rev. B 71, 125424 (2005).

[18] T. Fukuma, M. Kimura, K. Kobayashi, K. Matsushige, and H. Yamada, Rev. Sci. Instrum. 76, 053704 (2005).

[19] K. Kobayashi, H. Yamada, H. Itoh, T. Horiuchi, and K. Matsushige, Rev. Sci. Instrum. 72, 4383 (2001).

[20] T. Ichii, T. Fukuma, T. Yoda, K. Kobayashi, K. Matsushige, and H. Yamada, J. Appl. Phys. 107, 024315 (2010). 